\title{
Guide Wire Tracking During Endovascular Interventions
}

\author{
S.A.M. Baert, W.J. Niessen, E.H.W. Meijering, A.F. Frangi, and \\ M.A. Viergever \\ Image Sciences Institute, University Medical Center Utrecht \\ Rm E 01.334, P.O.Box 85500, 3508 GA Utrecht, The Netherlands \\ shirley@isi.uu.nl
}

\begin{abstract}
A method is presented to extract and track the position of a guide wire during endovascular interventions under X-ray fluoroscopy. The method can be used to improve guide wire visualization in the low quality fluoroscopy images. A two-step procedure is utilized to track the guide wire in subsequent frames. First a rough estimate of the displacement is obtained using a template matching procedure. Subsequently, the position of the guide wire is determined by fitting the guide wire to a feature image in which line-like structures are enhanced. In this optimization step the influence of the scale at which the feature is calculated and the additional value of using directional information is investigated. The method is applied both on the original and subtraction images. Using the proper parameter settings, the guide wire could successfully be tracked based on the original images, in 141 out of 146 frames from 5 image sequences.
\end{abstract}

Keywords: guide wire tracking, medical image processing, interventional radiology

\section{Introduction}

Endovascular interventions are rapidly advancing as an alternative for conventional invasive open surgical procedures. During interventions a guide wire is advanced under fluoroscopic control. Accurate positioning of the guide wire and the catheter with regard to the vasculature is a prerequisite for a successful procedure. Owing to the low dose used in fluoroscopy in order to minimize the radiation exposure of the patient and radiologist, image quality is often limited. Additionally, motion artifacts owing to patient motion and guide wire motion, further limit image quality. Therefore, a method to extract and track guide wires is presented which can deal with the low signal-to-noise ratio inherent to fluoroscopic images, and disappearance of the guide wire in a few frames owing to motion blur. The method can be used to improve guide wire visualization, potentially enabling a reduction in radiation exposure. It can also be used to detect the position of the guide wire in world coordinates for registration with preoperatively acquired images as a navigation tool for radiologists. 


\section{Methods}

In order to represent the guide wire, a spline parameterization is used. For all experiments in this paper, we used a third order B-spline curve defined by:

$$
\mathbf{C}(u)=\sum_{i=0}^{p} N_{i, 3}(u) \mathbf{P}_{i} \quad 0 \leq u \leq 1
$$

where $\mathbf{P}_{\mathbf{i}}$ denote the control points (forming a control polygon) with $p$ the number of control points and $N_{i, 3}(u)$ are the third degree B-spline basis functions defined on the non-periodic knot vector

$$
U=\left\{0, \ldots, 0, u_{4}, \ldots, u_{m-4}, 1, \ldots, 1\right\}
$$

where $m$ is the number of knots.

In order to find the spline in frame $n+1$ if the position in frame $n$ is known, a twostep procedure is introduced. First, a rigid translation is determined to capture the rough displacement of the spline. Next, a spline optimization procedure is performed in which the spline is allowed to deform for accurate localization of the guide wire. These steps can be understood as a coarse-to-fine strategy, where the first step ensures a sufficiently good initialization for the spline optimization.

\subsection{Rigid Transformation}

In order to obtain a first estimate of the displacement, a binary template is constructed based on the position in the present frame. The best location of this template in the new frame is obtained by determining the highest cross correlation with a certain search region in this image (or features derived from it; see section 2.3). This template matching technique is based on the assumption that a local displacement, $\mathbf{d}=\left(d_{x}, d_{y}\right)$, of a structure in one image $I_{0}$ can be estimated by defining a certain window $W$ (say $K \times L$ pixels in size) containing this structure, and by finding the corresponding window in a second image $I$ in the sequence by means of correlation. The correlation coefficient $(C C)$ is computed as:

$$
C C(\mathbf{d})=\frac{\sum_{\mathbf{x} \in W}\left[I_{0}(\mathbf{x})-\left\langle I_{0}\right\rangle_{W}\right]\left[I(\mathbf{x}+\mathbf{d})-\langle I\rangle_{W, \mathbf{d}}\right]}{\sqrt{\sum_{\mathbf{x} \in W}\left[I_{0}(\mathbf{x})-\left\langle I_{0}\right\rangle_{W}\right]^{2} \sum_{\mathbf{x} \in W}\left[I(\mathbf{x}+\mathbf{d})-\langle I\rangle_{W, \mathbf{d}}\right]^{2}}}
$$

where

$$
\left\langle I_{0}\right\rangle_{W}=\frac{1}{K L} \sum_{\mathbf{x} \in W} I_{0}(\mathbf{x}) \quad \text { and } \quad\langle I\rangle_{W, \mathbf{d}}=\frac{1}{K L} \sum_{\mathbf{x} \in W} I(\mathbf{x}+\mathbf{d})
$$

denote the mean values of the image intensities in the respective windows. 


\subsection{Spline Optimization}

After the first step is carried out, the spline is optimized under internal and external forces. The internal constraints are related to the geometry of the curve and influence the length (first derivative of the B-spline) and the bendedness (second derivative of the spline). This curvature $(K)$ is computed as:

$$
K=\int_{0}^{1} \frac{\left|\mathbf{C}^{\prime}(s) \times \mathbf{C}^{\prime \prime}(s)\right|}{L^{3}} d s
$$

where $L$ is the length of the curve given by

$$
L=\int_{0}^{1} \mathbf{C}^{\prime}(s) d s
$$

The parameters for the curvature are set sufficiently large to avoid strange shapes of the spline and sufficiently small to ensure that the internal forces only have a small influence on the total spline energy. For the external forces, the image intensity or a feature image derived form it (see section 2.3 is used. The spline contains four or five control points and one hundred sample points. The spline is optimized using Powell's direction set method [1].

\section{$2.3 \quad$ Feature Image}

It has previously been shown 2 that using original images for the matching and optimization steps, the guide wire could not effectively be tracked due to presence of other objects in the image. Therefore a feature image in which line-like structures are enhanced is used to determine the optimal spline position.

In order to enhance elongated structures in the image, the eigenvalues of the Hessian matrix are calculated at scale $\sigma$. This Hessian matrix is defined as:

$$
H=\left(\begin{array}{ll}
L_{x x} & L_{x y} \\
L_{x y} & L_{y y}
\end{array}\right)
$$

where $L_{x y}$ represents the convolution with the scaled Gaussian derivative

$$
L_{x y}=L * \frac{\partial^{2}}{\partial x \partial y} G(\mathbf{x}, \sigma)
$$

and $G(\mathbf{x}, \sigma)$ is given as

$$
G(\mathbf{x}, \sigma)=\frac{1}{2 \pi \sigma^{2}} e^{-\left(\frac{x^{2}+y^{2}}{2 \sigma^{2}}\right)}
$$

The corresponding eigenvalues are given by:

$$
\lambda_{1,2}(\mathbf{x}, \sigma)=\frac{1}{2}\left(L_{x x}+L_{y y} \pm \sqrt{\left(L_{x x}-L_{y y}\right)^{2}+4 L_{x y}^{2}}\right)
$$


Let $\lambda_{1}$ denote the largest absolute eigenvalue. On line-like structures $\lambda_{1}$ has a large output. Since we are interested in dark elongated structures on a brighter background, only positive values of $\lambda_{1}$ are considered; pixels with negative values of $\lambda_{1}$ are set to zero. The feature image is subsequently constructed by inverting this image since the optimization is based on a minimum cost approach, see Figure 1 Next to the eigenvalues, the eigenvectors give insight in the orientation
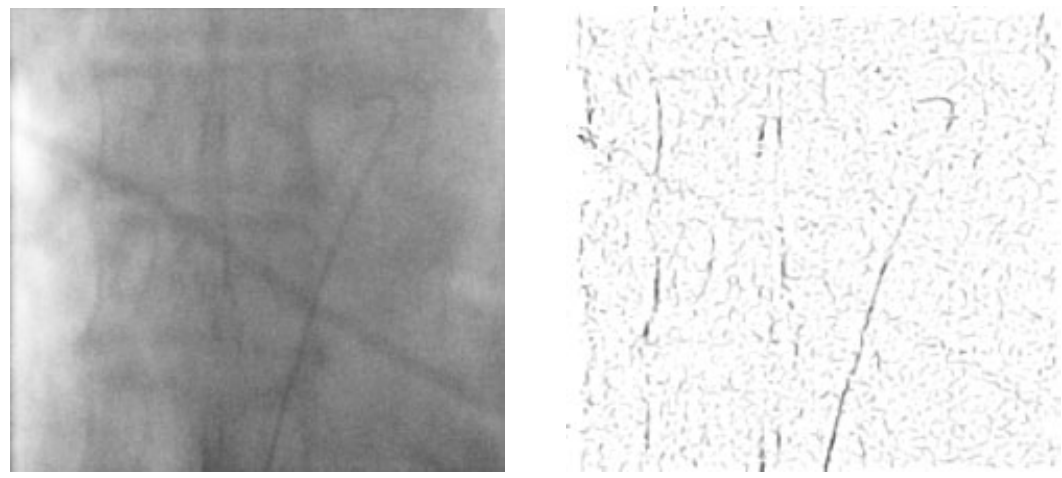

Fig. 1. The original image (left) and the feature image computed with the eigenvalues of the Hessian matrix with $\sigma=1.5$ (right).

of the line structure. Therefore, in the optimization scheme, we also investigated the use of this directional information to effectively attract the guide wire only to line structures with similar orientation. Hereto, the inner product between the spline and the orientation of the feature is used given by

$$
O\left(\hat{x}_{i}\right)=\lambda_{1}\left(\hat{e}_{2} \cdot \hat{x}_{i}\right)
$$

where $\hat{e}_{2}$ is the normalized eigenvector corresponding to $\lambda_{2}$ and $\hat{x}_{i}$ is the normalized first derivative of the spline in sample point $i$.

To enable a coarse-to-fine strategy and to reduce sensitivity to noise, the feature image can be calculated at multiple scales $(\sigma)$. We compare the use of the eigenvalue image calculated on the original image with computing the feature image on subtraction images. These images are obtained by subtracting the first frame from frame $n$. The first frame is used to ensure sufficient guide wire movement so as to make it clearly visible in the subtraction image. In our experiments we used image sequences with a maximum of 49 frames. For longer sequences it will be better to subtract for example frame $n-20$ from the present frame, to limit the effects of background motion. Alternatively, methods for motion correction in subtraction techniques can be used [3]. 


\subsection{Images}

The method was applied on five image sequences, three sequences of the thorax and two abdominal image sequences, with a sequence length between 14 and 49 frames. An overview of these sequences is given in Figure 2. Only J-tipped guide wires were used during the interventions. The image series were acquired on a H5000 (4 sequences) and a H3000 (1 sequence) X-ray fluoroscopy system (Philips Medical Systems, Best, the Netherlands).
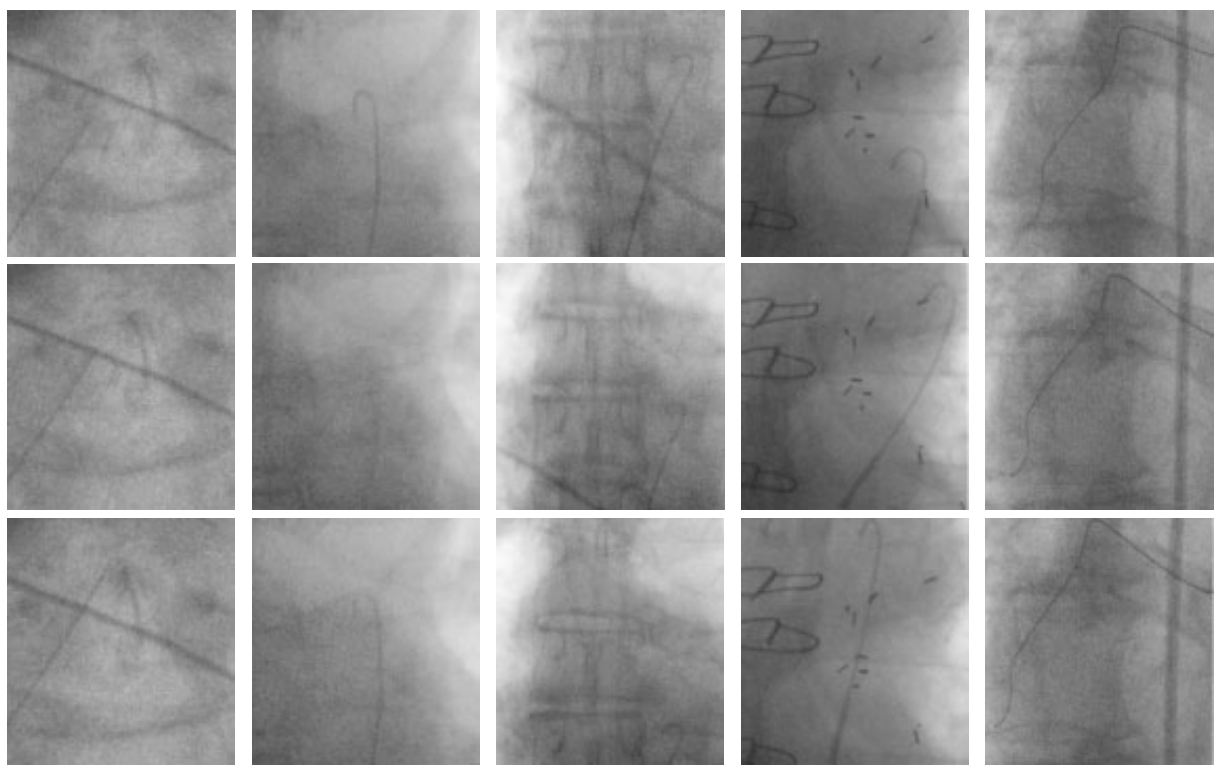

Fig. 2. Three non-subsequent frames of the sequences I to $V$ (left to right) used during the evaluation of the method.

\section{Results}

We compared the performance of the method on the Hessian feature image calculated on the original image with the one calculated on the subtraction image using different scales $(\sigma)$. In the spline optimization step, we compared the use of the scalar feature image and the vector feature image (see section 2.31). Evaluation of the tracking algorithm is difficult since no ground truth is available. In order to determine the effectiveness of the guide wire tracking procedure, the number of frames that could not be tracked was determined based on visual inspection of the results. When the spline model lies on top of the tip of the guide wire, the result was rendered as correct.

Table \summarizes the tracking results. Results without first applying a rough registration are not listed. These results were worse for every image sequence, which shows the need for a rough initialization before spline optimization. 
Table 1. Number of frames correctly tracked

\begin{tabular}{|c|c|c|c|c|c|c|c|}
\hline & & & Seq. I & Seq. II & Seq. III & Seq. IV & Seq. V \\
\hline \multirow{8}{*}{$\begin{array}{l}\text { Original } \\
\text { image }\end{array}$} & Hessian & $\sigma=1$ & $12 / 14$ & $24 / 25$ & $14 / 28$ & $28 / 30$ & $49 / 49$ \\
\hline & \multirow{7}{*}{ Orientation } & $\sigma=1.5$ & $12 / 14$ & $25 / 25$ & $25 / 28$ & $30 / 30$ & $49 / 49$ \\
\hline & & $\sigma=2$ & $9 / 14$ & $24 / 25$ & $23 / 28$ & $29 / 30$ & $49 / 49$ \\
\hline & & $\sigma=3$ & $1 / 14$ & $14 / 25$ & $9 / 28$ & $13 / 30$ & $2 / 49$ \\
\hline & & $\sigma=1$ & $12 / 14$ & $24 / 25$ & $17 / 28$ & $27 / 30$ & $49 / 49$ \\
\hline & & $\sigma=1.5$ & $12 / 14$ & $25 / 25$ & $25 / 28$ & $30 / 30$ & $49 / 49$ \\
\hline & & $\sigma=2$ & $10 / 14$ & $25 / 25$ & $22 / 28$ & $30 / 30$ & $49 / 49$ \\
\hline & & $\sigma=3$ & $2 / 14$ & $24 / 25$ & $3 / 28$ & $16 / 30$ & $38 / 49$ \\
\hline \multirow{8}{*}{$\begin{array}{l}\text { Subtraction } \\
\text { image }\end{array}$} & Hessian & $\sigma=1$ & $9 / 14$ & $18 / 25$ & $17 / 28$ & $23 / 30$ & $49 / 49$ \\
\hline & \multirow{7}{*}{ Orientation } & $\sigma=1.5$ & $9 / 14$ & $11 / 25$ & $20 / 28$ & $28 / 30$ & $49 / 49$ \\
\hline & & $\sigma=2$ & $8 / 14$ & $11 / 25$ & $22 / 28$ & $28 / 30$ & $48 / 49$ \\
\hline & & $\sigma=3$ & $2 / 14$ & $1 / 25$ & $22 / 28$ & $28 / 30$ & $48 / 49$ \\
\hline & & $\sigma=1$ & $9 / 14$ & $22 / 25$ & $14 / 28$ & $13 / 30$ & $49 / 49$ \\
\hline & & $\sigma=1.5$ & $10 / 14$ & $11 / 25$ & $16 / 28$ & $29 / 30$ & $49 / 49$ \\
\hline & & $\sigma=2$ & $11 / 14$ & $11 / 25$ & $20 / 28$ & $29 / 30$ & $48 / 49$ \\
\hline & & $\sigma=3$ & $1 / 14$ & $10 / 25$ & $20 / 28$ & $27 / 30$ & $48 / 49$ \\
\hline
\end{tabular}

Using the feature image computed on the original image for the registration and optimization step, we can observe from Table 1 that the scale $\sigma=1.5$ appears to be the optimal scale. With these settings, the guide wire could successfully be tracked in 141 out of 146 frames. In sequence I the tracking failed in 2 frames owing to line-like structures in the neighborhood of the guide wire. Motion blur due to a fast movement of the tip of the guide wire caused a mismatch in the registration step in 3 frames of sequence III. These failures appeared only in a single frame. Without manual intervention, the guide wire was tracked correctly in the subsequent frames. If other scales $(\sigma)$ are used during the eigenvalue computation, the performance of the tracking method becomes worse. For smaller $\sigma$, the tracking results, especially the matching step, is influenced by image noise. Conversely, at larger $\sigma$, broader line-like structures are more enhanced, so that the guide wire is not always detected correctly.

If we use orientation information instead of scalar information in the optimization step, the same results are obtained for $\sigma=1.5$. The failures on this scale were obtained in the matching step and they could not be corrected using different external energy forces in the spline optimization step. On a larger scale $(\sigma=3)$, the tracking results improved for sequence II and V. Using orientation information, the spline is less attracted to enhanced structures with an orientation that differs form the orientation of the spline. The optimization improves the localization of the spline which subsequently influences the registration step in the next frame.

If the eigenvalues of the Hessian matrix are calculated for the subtraction image, good results are obtained in the last two sequences, but the tracking failed most of the times in sequence I, II and III. These sequences are very noisy and by 
performing digital subtraction, the noise level is amplified. Using the orientation instead of scalar information in the optimization step for the subtraction images, the performance of the method hardly changes. An example of the good tracking results for sequence III and IV obtained with $\sigma=1.5$ is shown in Figure 3 and 4
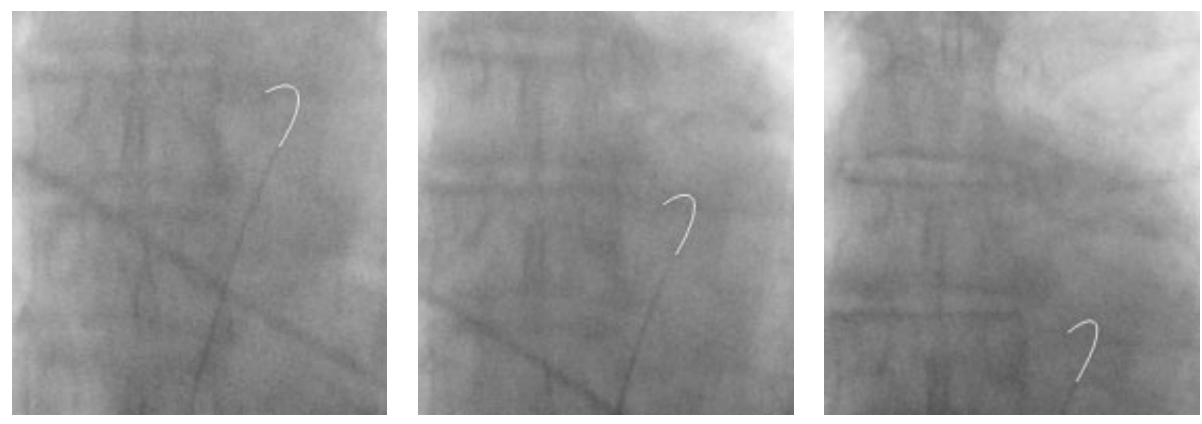

Fig. 3. Three frames (frame 2, 10 and 25) of sequence III, which gives an impression of the tracking results. The method was applied on the feature image in which the eigenvalues of the Hessian matrix were calculated with $\sigma=1.5$ on the original images of the sequence.

\section{Discussion}

There is relatively little literature on tracking guide wires from 2D fluoroscopy images. In [4], guide wire tracking is used to evaluate the possibility of extracting myocardial function from guide wire motion. However, guide wire tracking is only performed in a single frame and not in time. Some other work is aimed at the tracking of guide wires during endovascular interventions to control the position of a catheter inside the human body with external devices [56] (active tracking), or to reconstruct $3 \mathrm{D}$ catheter paths [7]. The presented method is a new approach to improve visualization and localization during endovascular interventions.

Based on the tests in the previous section, a number of conclusions can be drawn. First, the use of the cross-correlation step is helpful, as it supplies a good initialization for the subsequent fitting procedure. It is a known drawback from snake algorithms $[8$ to be sensitive to the initialization, and using the first rough alignment this is circumvented. Second, the scale parameter at which the feature image was computed is essential. It has to be selected sufficiently large to reduce sensitivity to noise, and sufficiently small to ensure that other line-like structures at a larger scale are not enhanced.

For the best parameter settings, the feature image containing the eigenvalues of the Hessian matrix gave the best overall results. The guide wire was successfully tracked in 141 out of 146 frames from 5 sequences. Subtracting a previous 

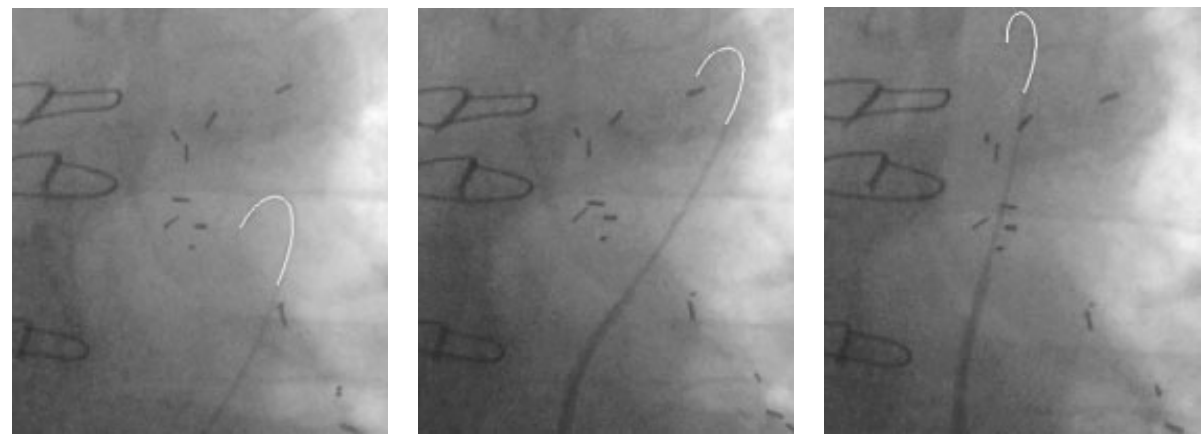

Fig. 4. Three frames (frame 4, 11 and 22) of sequence $I V$, which gives an impression of the tracking results. The method was applied on the feature image in which the eigenvalues of the Hessian matrix were calculated with $\sigma=1.5$ on the subtraction images of the sequence.

image from the current frame can help if there are elongated structures in the neighborhood of the spline, but the movement of the guide wire tip has to be large enough to get a good result. Using orientation information instead of scalar information in the optimization step gave some improvement on larger scales.

\section{References}

1. W.H. Press, S.A. Teukolsky, W.T. Vetterling, B.P. Flannery, Numerical Recipes in C: The art of scientific computing, 1992.

2. S.A.M. Baert, W.J.Niessen, E.H.W. Meijering, A.F. Frangi, M.A. Viergever, Guide wire tracking in interventional radiology, Proceedings of the 14th International Congress and Exhibition CARS 2000, 537-542.

3. E.H.W. Meijering, W.J. Niessen, M.A. Viergever, Retrospective Motion Correction in Digital Subtraction Angiography: A Review, IEEE Transactions on Medical Imaging 1999, Vol.18, No.1: 2-21.

4. D. Palti-Wasserman, A.M. Brukstein, R.P. Beyar, Identifying and Tracking a Guide Wire in the Coronary Arteries During Angioplasty from X-Ray Images, IEEE Transactions on Biomedical Engineering 1997, Vol.44, No.2: 152-164.

5. H. Starkhammar, M. Bengtsson, D.A. Kay, Cath-Finder Catheter Tracking System: a new device for positioning of central venous catheters. Early experiments from implantation of brachial portal systems. Acta Anaesthesiol. Scand. 1990, No. 34: 296-300.

6. J. Ragasa, N. Shan, R.W. Watson, Where antecubital catheters go: a study under fluoroscopic control, Anesthesiology 1989, No. 71: 378-380.

7. H.-J. Bender, R. Männer, C. Poliwoda, S. Roth, M. Walz, Reconstruction of 3D Catheter Paths from 2D X-ray Projections, Proceedings of the Second International Conference MICCAI'99: 981-989, Lecture Notes in Computer Science Vol.1679.

8. M. Kass, A. Witkin, D. Terzopoulos, Snakes: Active contour models. Int. Journal of Computer Vision 1987, Vol. 1, No. 4, 321-331. 